\title{
PANSHARPENING OF HYPERSPECTRAL IMAGES IN URBAN AREAS
}

\author{
Chembe Chisense, Johannes Engels, Michael Hahn and Eberhard Gülch \\ Stuttgart University of Applied Sciences \\ Schellingstr. 24 \\ D-70174 Stuttgart, Germany \\ johannes.engels@hft-stuttgart.de
}

Commission VII/6

KEY WORDS: image fusion, hyperspectral, resolution, urban, classification

\begin{abstract}
:
Pansharpening has proven to be a valuable method for resolution enhancement of multi-band images when spatially high-resolving panchromatic images are available in addition. In principle, pansharpening can beneficially be applied to hyperspectral images as well. But whereas the grey values of multi-spectral images comprise at most relative information about the registered intensities, calibrated hyperspectral images are supposed to provide absolute reflectivity values of the respective material surfaces. This physical significance of the hyperspectral data should be preserved within the pansharpening process as much as possible. In this paper we compare several common pansharpening methods such as Principal Component Fusion, Wavelet Fusion, Gram-Schmidt transform and investigate their applicability for hyperspectral data. Our focus is on the impact of the pansharpening on material classifications. Apart from applying common quality measures, we compare the results of material classifications from hyperspectral data, which were pansharpened by different methods. In addition we propose an alternative pansharpening method which is based on an initial segmentation of the panchromatic image with an additional use of map vector data.
\end{abstract}

\section{INTRODUCTION}

Pansharpening is a well-established technique for the enhancement of spatial image resolution, which allows the fusion of a low resolution multiband image with a high resolution panchromatic image. A mere upsampling of the multi-band image with an interpolation filter would result in a blurry quality with smoothed edges and missing short-wavelength constituents in the spatial Fourier expansions. Therefore at least the short-wavelength spatial information of the panchromatic image is integrated within fusion. This process, however, entails quite an amount of arbitrariness. Whereas the overall brightness of a pixel (apart from histogram matching) can be more or less directly adopted from the panchromatic information, the ratio of the grey values of the individual channels depends strongly on the respective pansharpening method.

More recently developed sensors suggest the application of pansharpening techniques, as those sensors frequently provide data in different resolution levels. Even more significant is the difference between hyperspectral sensors and colour cameras. Hyperspectral data usually feature a lower resolution than RGB images. However, pansharpening of hyperspectral imagery by a panchromatic image imposes two distinct problems:

- Often the "panchromatic" image does not comprise the full wavelength range of the hyperspectral image but only a part of it. The panchromatic image might e.g. be derived from RGB imagery, whereas the hyperspectral image covers a wider range from the visible up to the shortwave infrared. In such a case, the panchromatic image is not "representative", i.e. not a pixelwise average of the hyperspectral image.

- Calibrated hyperspectral data ideally feature reflectivity values of the respective material surfaces (disregarding dependencies on the source - reflector - sensor geometry, i.e. the
BRDF function). Therefore, in contrast to common imagery, an absolute hyperspectral grey value carries physical significance as a distinctive parameter of the material surfaces alone. Any "resolution enhancement" runs the risk of diluting this significance by a distortion of the parameter.

In the present paper we compare several common pansharpening methods with respect to their impact or suitability for hyperspectral data. We present in addition an alternative pansharpening method, which is based on the segmentation of the panchromatic image. The interpolation on the finer grid is performed by using data points from the same segment only. For the evaluation we apply visual inspection, profile analysis and some common quality measures. As more important, however, we rate a comparison of the classification results which are achieved from the pansharpened images.

\section{ESTABLISHED PANSHARPENING METHODS}

In the last three decades, a lot of algorithms have been developed which are, however, based on only few elementary principles. In any case, high-frequent panchromatic information is merged into spatially low resolution but spectrally differentiated data. We briefly outline the principles of some of the most common pansharpening methods. Here we follow the more detailed representation in (Pohl and van Genderen 1998) or (Hirschmugl et al. 2005).

\section{2-1 Gram-Schmidt Fusion}

The Gram-Schmidt Fusion works pixelwise. It was developed and described in detail by (Laben and Brower 2000). In a first step, a low resolution panchromatic channel is constructed as a weighted average of the original hyperspectral channels. Based on this first new channel, subsequently further linear combinations are formed by orthogonalization of the original bands with 
respect to their covariance as metric, exactly in the vein of the classical Gram-Schmidt-orthogonalization:

$$
\bar{G}_{\bar{i}}=\left(G_{i}-\mu_{i}\right)-\sum_{k=1}^{i-1} \frac{C_{i, \bar{k}}}{C_{\bar{k}, \bar{k}}} \bar{G}_{\bar{k}}
$$

Here $G_{i}$ denotes the greyvalue of an individual pixel in the original channel $i, \bar{G}_{\bar{k}}$ the greyvalue of the same pixel in the (transformed) Gram-Schmidt channel $\bar{k} . \mu_{i}$ is the mean greyvalue of channel $i$ taken over all pixels. The covariance $\mathbf{C}_{i, j}$ between two original channels $i, j$ is empirically determined by

$$
C_{i, j}=\frac{\sum_{\text {Pixels }}\left(G_{i}-\mu_{i}\right)\left(G_{j}-\mu_{j}\right)}{N}
$$

where $N$ denotes the total number of pixels. Due to construction, the Gram-Schmidt channels are all uncorrelated.

The histogram of the high-resolving panchromatic image is matched to the histogram of the artificial, low-resolving panchromatic image. In this way at least the global (if not the local) grey value distribution of the panchromatic image is adjusted to the intensity distribution of the hyperspectral data. Then, the low-resolution panchromatic is replaced by the high resolution panchromatic, the remaining hyperspectral channels are upsampled and adopted. Finally, the Gram-Schmidt transform is inverted.

\section{2-2 PCA Fusion}

This method resembles very much the Gram-Schmidt Fusion. In contrast to the latter, the "artificial" low resolution panchromatic channel is constructed as that linear combination of all bands, which corresponds to the maximal eigenvalue of the principal component analysis. The eigenvectors of the PCA are by construction orthogonal; the corresponding combination bands are uncorrelated. The further processing is the same as with the Gram-Schmidt Fusion.

Obviously, PCA Fusion is based on the assumption that the panchromatic image corresponds best with the linear combination of bands which features the highest variance. As in the case of the Gram-Schmidt Fusion, a global histogram matching is employed.

\section{2-3 Wavelet Fusion}

Wavelet Fusion is based on the concept of image pyramids, see e.g. (Ranchin and Wald 2000). Given a high resolution image, the base of the pyramid is just the image itself, whereas the higher levels of the pyramid consist in successive approximations, i.e. each level consists in an approximation of the previous level. In a second pyramid the difference images between consecutive approximations are represented. For the purpose of pansharpening, the high resolution panchromatic image is approximated successively until the resolution of the hyperspectral data is reached. Then for each individual channel, the approximation of the panchromatic image is replaced by the respective hyperspectral data. High resolution images are constructed by successively adding the difference images of the panchromatic image to each individual channel.

Whereas many approximation methods are imaginable for this process, the Discrete Wavelet Transform constitutes a particularly elegant and - in view of the famous Mallat algorithm - also efficient possibility, see e.g. (Mallat 2009). The workflow of the wavelet fusion reminds to the workflow of PCA Fusion. For the former method, however, the transformations (forward and inverse wavelet transform) act on the panchromatic image, whereas for the latter the transformations (forward and inverse PCA) work on the hyperspectral data.

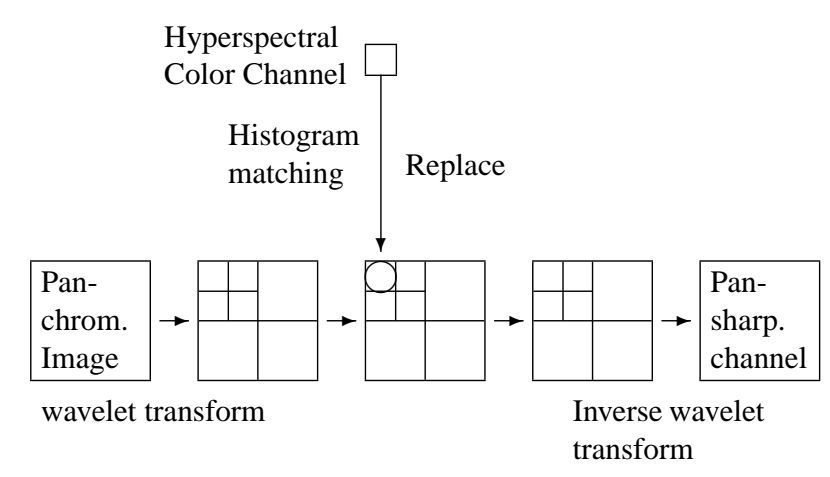

Figure 1: Workflow of Wavelet Fusion according to (Hirschmugl et. al 2005)

Different from PCA and Gram-Schmidt Fusion, only local variations of the panchromatic image affect the pansharpening result. This is due to the fact that only the wavelet coefficients (i.e. the difference images) of the panchromatic image remain, whereas the approximation coefficients of the low resolution level are replaced by the corresponding coefficients of the respective hyperspectral channel.

\section{A SEGMENTATION-BASED PANSHARPENING METHOD}

In a common RGB image the absolute grey and color values may differ noticeable from exposure to exposure. Hyperspectral data in contrast are usually calibrated in such a way, that the grey values represent the absolute reflectivity of the respective surface materials for the respective wavelength, i.e. the ratio between reflected and incident radiation. The reflectivity is a property of the surface material alone; it is of particular value for the distinction of surface materials. Therefore we aim at a pansharpening method which respects as much as possible the original hyperspectral data. In a first step, we perform a segmentation of the high resolution panchromatic image. The segments are assumed to be homogeneous, ideally each segment should feature one material only. In a second step, the hyperspectral data are interpolated by means of an inverse distance method on the finer grid. Hereby only such pixels of the original hyperspectral image are used, which are located completely in the same segment as the interpolation position.

\section{3-1 Segmentation of the Panchromatic Image (RGB-image)}

The segmentation of the panchromatic image was performed by the well-known eCognition software, see (eCognition User Guide). This software enables the simultaneous processing of multiple channels in a hierarchical way. eCognition successively aggregates pixels with similar grey values to segments. Criteria for the aggregation of adjacent objects are on the one hand the heterogeneity of the grey values of the combined object, on the other hand its geometric form. These criteria can be balanced by three parameters: "scale", "color" and "shape". Adequate parameters have to be selected by trial and error. Focused on application in urban areas, in most cases a one-to-one relation between segments and building regions will be optimal, though roofs also may consist of different materials.

As for our test area RGB orthophotos and in addition ALK vector data (parcels, buildings) were available, the segmentation was performed hierarchically. The borders as given by the vector data already imply a segmentation. This segmentation was refined by 
the orthophotos in the sense of a further subdivision; i.e. the borders defined by the vector data are not changed, only additional borders introduced. For the parameters "scale", "shape", "compactness" the values $100,0.2$, and 0.9 were selected.

\section{3-2 Interpolation of the Hyperspectral Channels on the Finer Grid by Inverse Distance Interpolation}

For the interpolation on the finer grid we have chosen the Inverse Distance Method. This method on the one hand requires less computational effort than e.g. Minimum Curvature Interpolation, on the other hand avoids undesired oscillations as might appear with Kriging or Linear Prediction. The pixel centers of the hyperspectral image are considered as data points, whereas the pixel centers of the panchromatic grid (finer resolution) are considered as interpolation points. The workflow of the interpolation is as follows:

We run through all the segments and determine for each segment $S$ a list of pixels of the finer grid which are contained in $S$; the centers of these pixels define the interpolation points. In addition, we select all the pixels of the coarser grid which are completely contained in $S$ - they will give us the data points. The Inverse Distance Interpolation for any interpolation point and channel is performed according to the formula

$\widehat{y}_{i}=\sum_{k=1}^{K} \frac{y_{k} w_{i k}}{\sum_{l} w_{i l}}$ where $w_{i k}=\sqrt{\left(r_{i}-r_{k}\right)^{2}+\left(c_{i}-c_{k}\right)^{2}+\alpha}$

Here $\mathbf{y}$ denotes the observation vector, i.e. the grey values of the data pixels for the respective channel, $\alpha$ is a regularizing parameter, $K$ is the number of data points within the segment. $r_{i}, r_{k}, c_{i}, c_{k}$ are the row and column indices of the interpolation or data point with respect to the finer grid.

Higher powers of the distance are common for Inverse Distance Interpolation. As our data are somewhat noisy, we appreciate the smoothing effect within the segments due to the low power 1 of the distance.

For many small segments no pixel in the low resolution was encountered, which was located completely inside the segment. For such cases all grey values in the pansharpened image were set to the corresponding values of the original hyperspectral image.

\section{TEST DATA}

For the evaluation of the pansharpening methods we use hyperspectral data of Ludwigsburg, Germany. The data was recorded on August, 202010 with the HyMap sensor within the annual HyEurope-campaign of the German center of aviation and space flight (DLR); it comprises 125 channels in the range of $0.45-$ $2.49 \mu \mathrm{m}$. The ground resolution is $4 \mathrm{~m}$. The test area consists in 6 strips with an overall extension of $9.6 \times 9.0 \mathrm{~km}^{2}$. The panchromatic image was derived from an RGB-orthophoto which was registered in the spring of 2010. The ground resolution of this orthophoto amounted originally to $25 \mathrm{~cm}$; it was degraded to $1 \mathrm{~m}$ in order to keep the pansharpening factor moderate.

\section{EVALUATION OF THE PANSHARPENING METHODS}

\section{5-1 General Visual Impression}

The most obvious criterion for the quality of a pansharpening result is the visual impression. This criterion might be misleading in some cases, hoewever, human vision is quite perceptive for image sharpness and color differences. Figures $2-6$ show the RGB orthophoto, RGB-channels of the original HyMap data and the pansharpened data, achieved with three different methods, for a small region in the downtown in Ludwigsburg. In the lower left corner the station and some railway tracks are visible. As the results of the PCA Fusion and the Gram-Schmidt Fusion can hardly be distinguished with bare eyes, only one of them is reproduced.

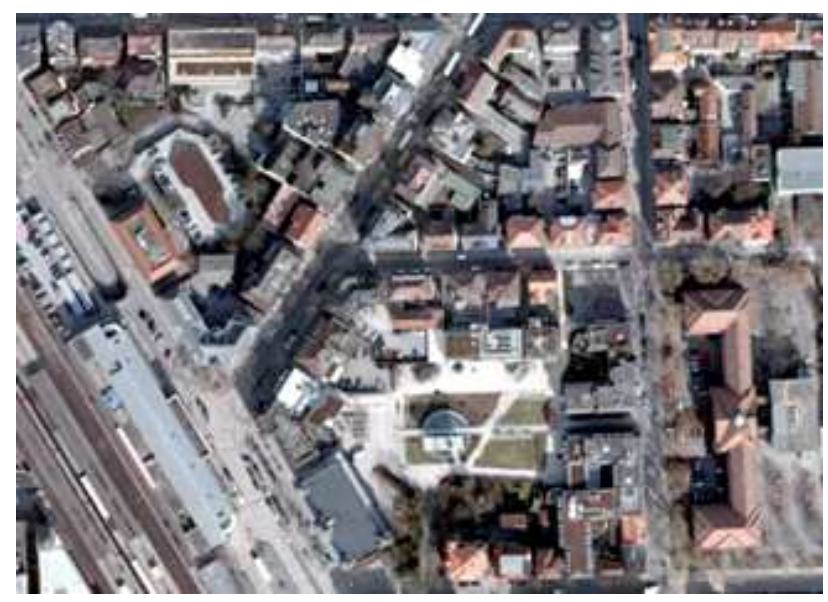

Figure 2: Original RGB-orthophoto

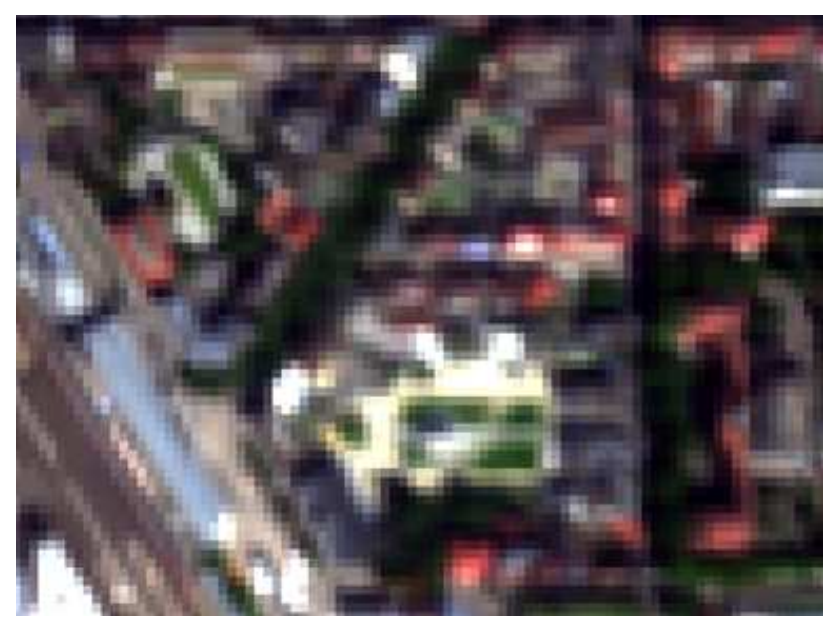

Figure 3: RGB channels of the original hyperspectral image

At first glance, unambiguously the result of the Gram-Schmidt Fusion looks best. The Wavelet Fusion shows some undesired "ghost-" or "staircase-" artefacts. This phenomenon is also reported by (Hirschmugl et al. 2005); it might be due to the combination of different spatial wavelength ranges from different images. A sharp edge in the space domain corresponds to strong short-wave components in the frequency domain with well-defined ratios between amplitudes of different wavelengths. If long- and short-wave components are assembled from different data sources, these ratios might be distorted, which results at best in unsharp edges, at worst in "staircase-" or even oscillation effects, so that the edge appears to be "echoed". The segmentation-based pansharpening on the other hand features a pronounced terrace- or "sycamore-bark"-like pattern; the edges clearly reflect the segmentation borders. Whereas the visual impression might be unsatisfactory, this "sharpening" of edges turns out to be beneficial when it comes to classification. 


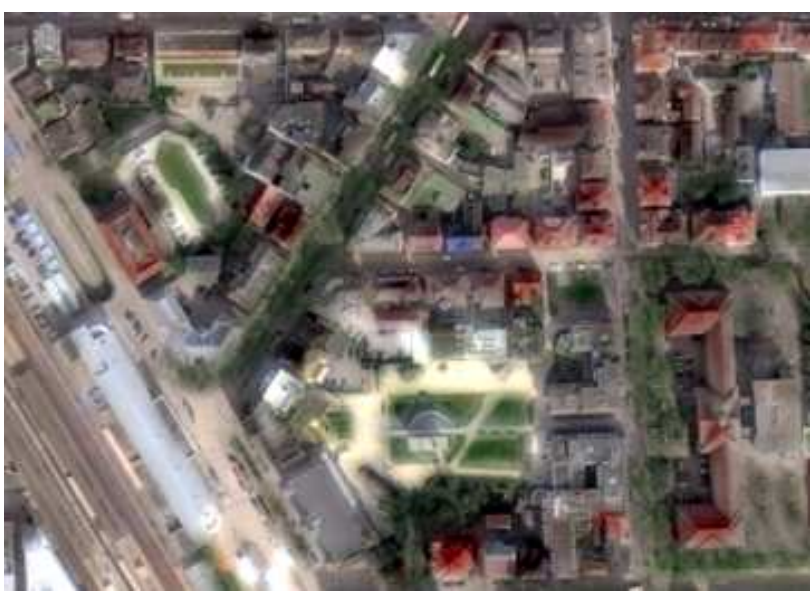

Figure 4: RGB channels of the Gram-Schmidt Fusion image

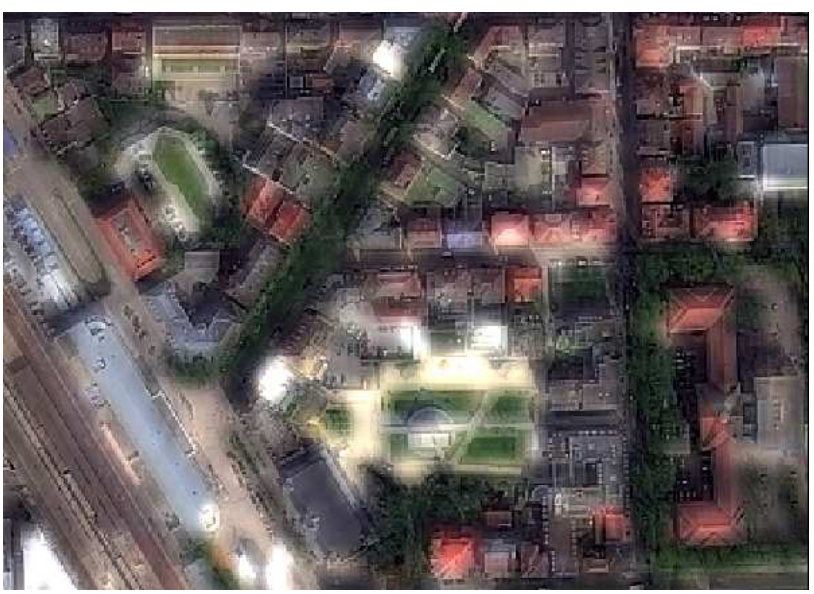

Figure 5: RGB channels of the Wavelet Fusion image

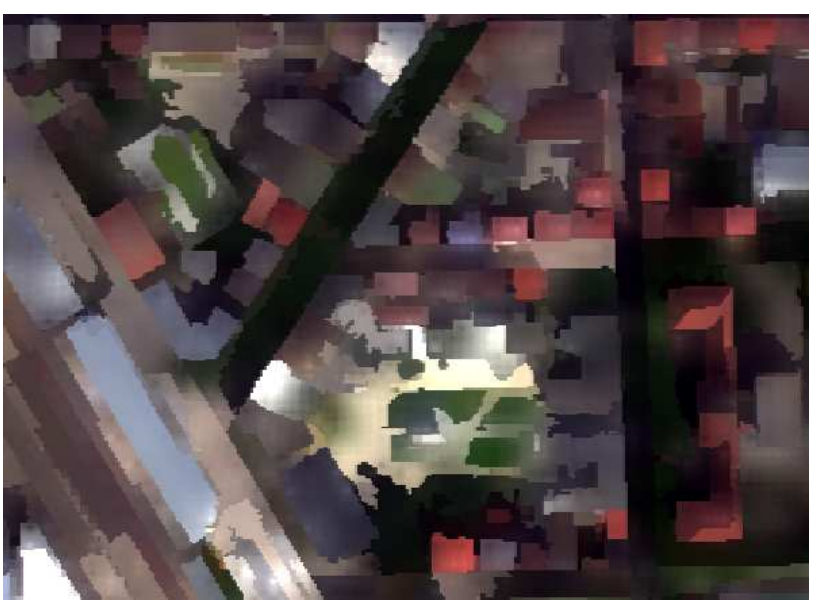

Figure 6: RGB channels of the Segmentation-based Fusion image

\section{5-2 Profiles}

The progression of greyvalues along profiles represents a valueable criterion for the evaluation of pansharpening methods. Particularly critical are profiles where the visible and infrared channels strongly diverge. (Hirschmugl et al. 2005) report for some fusion methods even trend reversals in the greyvalue progression.

In Figures 7, 8 we consider two profiles in the original HyMap data and three pansharpening methods. For each profile of a pansharpened image we represent three visible (RGB) and one infrared channels, viz. $454 \mathrm{~nm}, 544 \mathrm{~nm}, 635 \mathrm{~nm}$ and $1500 \mathrm{~nm}$ wavelengths. The infrared channel is represented in pink in each case.

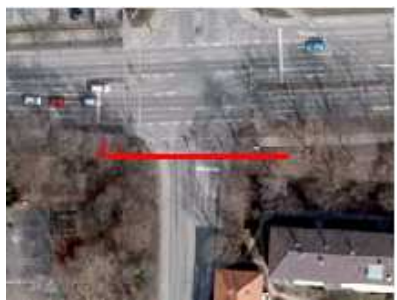

(a) Location of Profile 1 in the RGB image

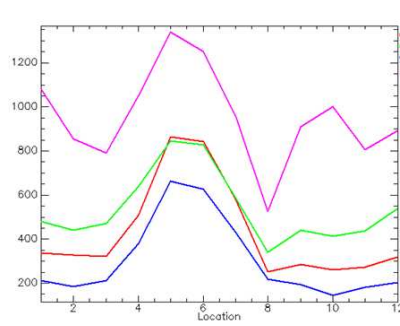

(c) Profile of the original hyperspectral image

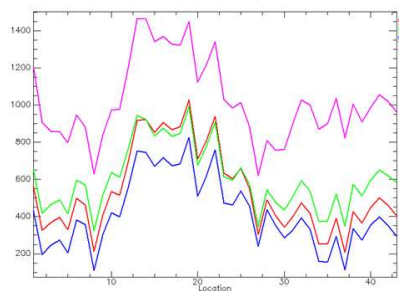

(e) Profile of the Wavelet Fusion (f) Profile of the Segmentationimage

Figure 7: Profile 1 for different Fusion Methods

The first profile (Figure 7) extends over a street which is on the left side seamed by small bushes and by trees at the right. As the RGB and the hyperspectral data have been registered at different seasons, the respective profiles show a different behaviour: The RGB image was registered at early springtime, so there was only little foliation on the trees; the corresponding profile shows a gradual decay from left to right. The hyperspectral data were recorded in summer, the foliation was fully developed and the trees overhanging to the street; the profile shows a more abrupt decay already in the middle of the road. In particular the GramSchmidt Fusion, but to a lower extent also the Wavelet Fusion reflects the behaviour of the RGB image, because the overall brightness is (completely or partly) adapted from there. The profile of the segmentation-based method, on contrary, features sharper edges between road and vegetation; as the vector data reflect the actual border of the road on the ground, the segment extends further to the right than the greyvalues in the images suggest, and the low power of the inverse distance interpolation, which gives relative high weight to far data points, provides an extension of the characteristic "road signature" to the right. Gram-Schmidt and Wavelet fusion feature both strong oscillations which are obviously also inherited from the RGB image and which affect all four channels in the same way; the segmentation-based interpolation on contrary exhibits a strong smoothing effect within the segments. 
The absolute values of the Wavelet Fusion and the Segmentationbased Fusion are relatively close to the original HyMap data, whereas the absolute values of the Gram-Schmidt Fusion exceed the original ones by about $50 \%$. This is evidently due to the fact that the Gram-Schmidt Fusion adapts the overall brightness to the panchromatic image (histogram matching is only performed globally), whereas the Wavelet Fusion introduces mostly shortwave components of the panchromatic image to the pansharpened image.

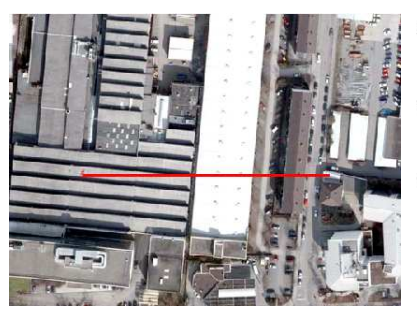

(a) Location of Profile 2 in the RGB image

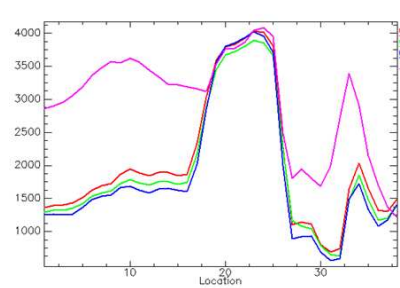

(c) Profile of the original hyper- (d) Profile of the Gram-Schmidt Fuspectral image

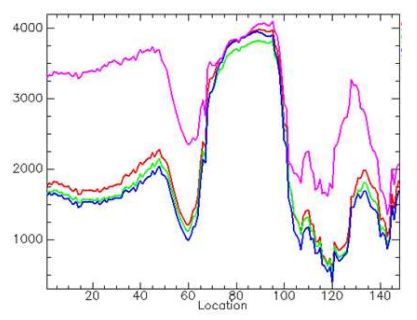

(e) Profile of the Wavelet Fusion (f) Profile of the Segmentationimage

Figure 8: Profile 3 for different Fusion Methods

Profile 2 (Figure 8 ) is of special interest due to the different behaviour of the infrared channel in the Gram-Schmidt and the Wavelet Fusion. The small dark strips between the inclined panels (shadowy areas) on the left building are too small to be resolved in the hyperspectral image, whereas they do appear in the panchromatic image. The depths of the corresponding sinks in the Wavelet Fusion are more or less independent of the channel, whereas for the Gram-Schmidt Fusion they appear to be proportional to the "continuum" level of the respective channel. The Segmentation-based method exhibits its "generalizing" tendency again.

\section{5-3 Quality Measures for the Comparison of the original and the pansharpened data}

Some authors propose quality measures based on the differences between the original or upsampled hyperspectral data, respectively, and the pansharpened data. Here we evaluate the root mean square error, correlation coefficients and the universal quality index proposed by (Wang and Bovik 2002).

\section{Root mean square error:}

The root mean square error is used to quantify the average amount of distortion in each pixel of the pansharpened images. The root mean square is computed between the original hyperspectral image (resampled to the resolution of the pansharpened hyperspectral data) and the pansharpened hyperspectral images. The results are shown in Table 1:

\begin{tabular}{|l|l|l|l|l|l|l|}
\hline & R & G & B & I1 & I2 & I3 \\
\hline PCA & 465 & 365 & 351 & 821 & 836 & 870 \\
Gram-Schmidt & 457 & 375 & 349 & 787 & 800 & 804 \\
Wavelet & 269 & 243 & 246 & 369 & 375 & 484 \\
Seg.-based & 231 & 195 & 193 & 365 & 371 & 490 \\
\hline
\end{tabular}

Table 1: Root mean square error of different fusion methods compared to the upsampled original hyperspectral image (reflectivity values, range $0-10000$ )

The wavelengths of the represented bands are $0.635 \mu \mathrm{m}(\mathrm{R}), 0.544$ $\mu \mathrm{m}$ (G), $0.454 \mu \mathrm{m}$ (B), $1.50 \mu \mathrm{m}$ (I1), $1.805 \mu \mathrm{m}$ (I2) and $2.485 \mu \mathrm{m}$ (I3). It is obvious that for the most wavelengths the grey values of the Segmentation-based Fusion are least distorted.

\section{Correlation Coefficients:}

Table 2 compares the correlations between different channels and the panchromatic image. PCA and Gram-Schmidt Fusion show the highest correlation values, which means that for these two methods the contribution of the panchromatic image is the highest. Particularly high are the correlation coefficients with the three infrared channels. On the opposite, the Segmentation-based Fusion image is closer to the original hyperspectral image which is desirable as the differences between the individual channels are levelled out to a lower extent.

\begin{tabular}{|l|l|l|l|l|l|l|}
\hline & $\mathrm{R}, \mathrm{P}$ & $\mathrm{G}, \mathrm{P}$ & $\mathrm{B}, \mathrm{P}$ & $\mathrm{I} 1, \mathrm{P}$ & $\mathrm{I} 2, \mathrm{P}$ & $\mathrm{I} 3, \mathrm{P}$ \\
\hline Original data & 0.53 & 0.51 & 0.48 & 0.46 & 0.44 & 0.33 \\
\hline PCA & 0.80 & 0.75 & 0.70 & 0.97 & 0.98 & 0.82 \\
Gram Schmidt & 0.79 & 0.76 & 0.70 & 0.93 & 0.93 & 0.74 \\
Wavelet & 0.69 & 0.68 & 0.65 & 0.61 & 0.59 & 0.51 \\
Seg.-based & 0.52 & 0.49 & 0.47 & 0.43 & 0.41 & 0.33 \\
\hline
\end{tabular}

Table 2: Correlation coefficients between the panchromatic image and different bands of the original and the pansharpened images

\section{Universal Quality Index:}

Quite common is the Universal Image Quality index $Q$ given by (Wang and Bovik 2002):

$$
Q:=\frac{4 \sigma_{x y} \bar{x} \bar{y}}{\left(\sigma_{x}^{2}+\sigma_{y}^{2}\right)\left[\bar{x}^{2}+\bar{y}^{2}\right]}=\frac{\sigma_{x y}}{\sigma_{x} \sigma_{y}} \cdot \frac{2 \bar{x} \bar{y}}{\bar{x}^{2}+\bar{y}^{2}} \cdot \frac{2 \sigma_{x} \sigma_{y}}{\sigma_{x}^{2}+\sigma_{y}^{2}}
$$

Here $x=\left\{x_{i} \mid i=1,2, \cdots, N\right\}, y=\left\{y_{i} \mid i=1,2, \cdots, N\right\}$ denote the original and test image signals, respectively, $i$ is the pixel index. $Q$ can be applied to each channel individually. As the last term in the defining formula shows, $Q$ can be decomposed into three factors which comprise a) the correlation coefficient (correlation between the two images), b) a similarity measure between the arithmetic means $\bar{x}$ and $\bar{y}$ and c) a similarity measure between the standard deviations $\sigma_{x}$ and $\sigma_{y}$.

The "optimal" $Q$ value of 1 e.g. is achieved if the images $x$ and $y$ 
are identical. Table 3 shows that by this criterion the Segmentationbased Fusion has the highest rank.

\begin{tabular}{|l|l|l|l|l|l|l|}
\hline & $\mathrm{R}$ & $\mathrm{G}$ & $\mathrm{B}$ & $\mathrm{I} 1$ & $\mathrm{I} 2$ & $\mathrm{I} 3$ \\
\hline PCA & 0.26 & 0.27 & 0.27 & 0.18 & 0.17 & 0.23 \\
Gram Schmidt & 0.26 & 0.26 & 0.27 & 0.19 & 0.19 & 0.26 \\
Wavelet & 0.49 & 0.34 & 0.32 & 0.45 & 0.15 & 0.42 \\
Seg.-based & 0.51 & 0.50 & 0.50 & 0.50 & 0.50 & 0.25 \\
\hline
\end{tabular}

Table 3: Universal Quality Index according to (Wang and Bovik 2002) for different channels of the pansharpened images

\section{5-4 Classification Results}

As we use hyperspectral data mostly for material classication, we prefer to compare the pansharpening methods with respect to the result of a supervised classification which they provide. Here we present only first results of an ongoing research.

Before classifying the different pansharpened images, training regions for different surface materials were defined. We have used 18 training regions for 12 roof materials plus vegetation. In order to determine the accuracy of the classification, the results are compared with ground truth information. As test regions for the evaluation we use the training regions plus 33 additional regions. The ground truth has to be improved in the future, however.

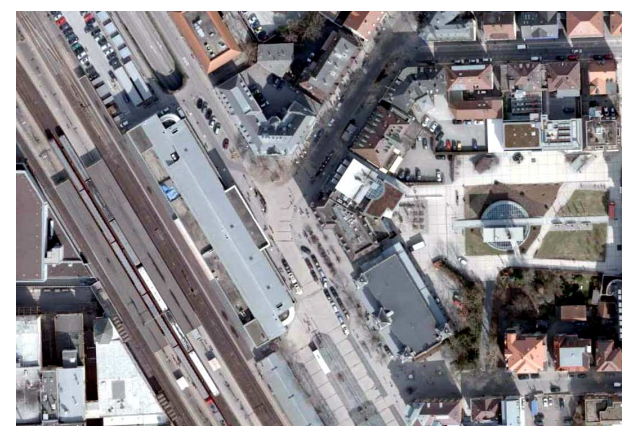

(a) RGB Image

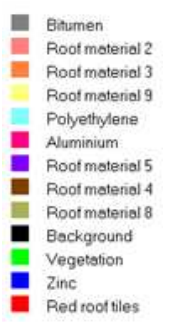

(b)

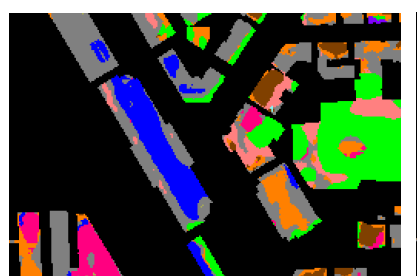

(c) PCA Fusion

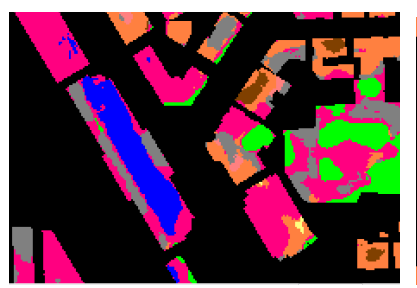

(e) Wavelet Fusion

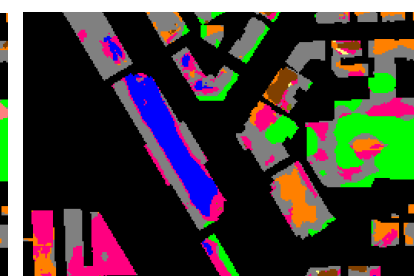

(d) Gram-Schmidt Fusion

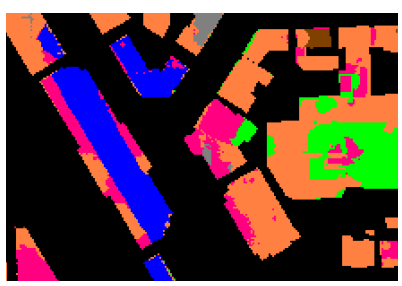

(f) Segmentation-based Fusion
Figure 9: Classification Example

Figures 9 gives an impression about the results of a SAM classification. As expected, the Segmentation-based Fusion yields a good homogeneity of the segments. More significant are the evaluation measures which are reported in Table 4. The Overall Accuracy gives the ratio between correctly classified pixels and the total number of pixels. The so-called kappa coefficient additionally takes into account possible chance correct classifications (Cohen 1960). The results in Table 4 favour the Wavelet and the Segmentation-based Fusion - inspite of their minor visual quality.

\begin{tabular}{|l|l|l|}
\hline Fusion & $\kappa$ & $\begin{array}{l}\text { Overall } \\
\text { Accuracy }\end{array}$ \\
\hline PCA & 0.70 & $74.7 \%$ \\
Gram Schmidt & 0.72 & $76.7 \%$ \\
Wavelet & 0.82 & $85.3 \%$ \\
Seg.-based & 0.80 & $84.0 \%$ \\
\hline
\end{tabular}

Table 4: Accuracy Measures of the Classification Results based on different Fusion Methods

\section{CONCLUSIONS AND FUTURE WORK}

Pansharpening in general not only affects the overall brightness of hyperspectral data, but also the shape of the hyperspectral signatures, which is important for material classification. In order to distort the original data as few as possible, we propose to perform in a first step a segmentation of the panchromatic image, employing map vector data in addition if available. In a second step the data are resampled on the finer grid, whereby the interpolation is performed only by means of data pixels which are located completely in the respective segment. A comparison of different pansharpening methods reveals very different rankings dependent on the quality criteria. We think that our method has a good potential for the pansharpening for classification purposes. Further investigations will be based on better ground truth information and more sophisticated classification methods. They should also comprise a more detailed scrutiny of misclassified regions.

\section{REFERENCES}

Cohen, J., 1960. A coefficient of agreement for nominal scales. Educational and Psychological Measurement, 20, 37-46

Hirschmugl, M., Gallaun, H., Perko, R., and Schardt, M. 2005: "Pansharpening"-Methoden für digitale, sehr hoch auflösende Fernerkundungsdaten. In: Beiträge zum 17. AGIT Symposium, Salzburg, Austria, July 06-08 2005.

Laben, C.A. and Brower, B.V. 2000: Process for enhancing the spatial resolution of multispectral imagery using pansharpening. United States Eastman Kodak Company (Rochester, New York). US Patent 6011875.

Mallat, S., 2009. A Wavelet Tour of Signal Processing. Elsevier Inc.

Pohl, C. and van Genderen, J.L. 1998: Multisensor image fusion in remote sensing: concepts, methods and applications. International Journal of Remote Sensing, vol.19, no.5, pp.823- 854, Mar. 1998.

Ranchin T., Wald L., 2000: Fusion of high spatial and spectral resolution images: the ARSIS concept and its implementation. Photogrammetric Engineering and Remote Sensing, 66(1), 49-61

Wang, Z. and Bovik, A. 2002: A Universal Image Quality Index. IEEE Signal Processing Letters, vol. 9 no. 3 p. 81-84 2002

Ecognition User Guide http://www.gis.unbc.ca/help/software/ecognition4/ELuserguide.pdf visited in April 2012 